\title{
475775 - HEADACHE,DIPLOPIA,NUMBNESS-CAN AIR DO ALL THIS?
}

\author{
Tennielle Gofton, $\mathrm{MD}^{3}$, Amna Masood, BSC,MBBS,MD ${ }^{1}$, Craig Railton, \\ BSC,Phd,MD,FRCPC ${ }^{2}$ \\ 1. Anesthesiology, University of Western Ontario, London, ON, Canada \\ 2. Neurology, University of Western Ontario, London, ON, Canada \\ 3. Anesthesiology and Perioperative Medicine, University of Western Ontario, \\ London, ON, Canada
}

Purpose: Epidurals are widely used in the management of pain in labor and delivery. Although dural puncture headaches affects approximately one in three hundred patients more serious neurologic complications are rare. We present a case of an 18 year old primigravida who requested an epidural and developed some very rare complications. We will show imaging and review some of the known cases of patients that developed similar symptoms and review management strategies for low pressure syndromes due to epidural complications.

Clinical Features: Clinical features: Patient consent was obtained. An 18 year old, primigravida was treated with Fragmin for a deep vein thrombosis which developed at 27 weeks gestation. She presented in premature labor at 34 weeks gestation. She requested an epidural after 25 hours of her last Fragmin dose. An uneventful epidural at L3-4 was placed and several hours later delivered a healthy female. The following morning she awoke with a fronto-occipital headache, left sided T5-T8 numbness and neck pain. The headache eventually waned, and was discharged by the obstetric team with an advice to use acetaminophen. On post delivery day four she was admitted for worsening symptoms. Anesthesia,Neurology, and spine surgery were urgently consulted. She underwent MRI head and spine: the imaging showed epidural air at multiple levels. The patient was given a diagnosis of possible post dural puncture headache with nerve root irritation secondary to epidural air. She was managed conservatively. She returned on Day 12 with severe rightward gaze diplopia and a general worsening of symptoms. She was admitted and repeat imaged by MRI. There was no evidence of epidural air but meningeal enhancement was observed. Low pressure syndrome was diagnosed and the patient was treated with an epidural blood patch. Her diplopia improve over twenty-four hours and her other symptoms resolved over the next few weeks. A literature search of Medline and Pub Med was done to find similar cases and treatment strategies. The literature search found six case reports and several management strategies. We will summarize and present the results of the literature search.

Conclusion: Inadvertent unrecognized dural tap can often have rare and unusual presentation such as diplopia. The development of a chronic low pressure syndrome following a dural tap is rare and can create treatment dilemmas.

References: 1 . Gielen M. Post dural puncture headache (PDPH): a review. Regional Anesthesia 1989; 14(3)\& 101-6. Volume 24, Issue 5, September-October 1999, Pages 470-472 2. www.cja-jca.org/cgi/content/full/54/2/1463. 3.Neurol Clin N Am 22 (2004) 55- 74Anaesthesia, 2006, 61, pages 370-375 2006 